\title{
A MANUFACTURING PLANNING AND CONTROL SYSTEM FOR A FLEXIBLE MANUFACTURING SYSTEM
}

\author{
J. SLOMP and W. H. M. ZIJM \\ Laboratory of Production Management and Operations Research, Department of Mechanical Engineering, \\ University of Twente, P.O. Box 217, 7500 AE Enschede, The Netherlands
}

\begin{abstract}
A flexible manufacturing system (FMS) can offer important advantages in terms of more efficient production, shorter throughput times, lower stocks and a higher quality of work. To realize these advantages, a well designed manufacturing planning and control system (MPCS) is a key condition. In practical cases, these planning and control systems are often based on a hierarchical decision structure. This paper discusses the most relevant characteristics of such a hierarchical MPCS and, by using a practical case, describes possible solutions to a variety of subproblems.
\end{abstract}

\section{INTRODUCTION}

A flexible manufacturing system (FMS) is an integrated manufacturing system consisting of automated workstations linked by a material handling system capable of processing different jobs simultaneously. Its effective use in a particular situation is largely determined by its degree of efficiency, flexibility and multifunctionality.

\section{Efficiency}

In an FMS, all the preparatory work, like the clamping of parts on pallets/fixtures and the presetting of tools, can be done during machining time. This results in an almost complete absence of changeover times while processing different parts sequentially. An important aspect of efficiency furthermore concerns the capability of an FMS to operate during an unmanned night shift. However, the efficiency of an FMS is limited. Often, the buffer storage of pallets in the FMS is not large enough to support a fully unmanned night shift. A balanced workload is also an important condition of an efficient operation of the FMS.

\section{Flexibility}

Due to the presence of all essential equipment in the system, like pallets, fixtures, tools and NC programs, and through the absence of changeover times, an FMS can easily change from the processing of one mix of jobs to another mix. However, the flexibility of the system is limited by the availability of equipment (e.g. a unique cutting tool cannot be used simultaneously on different machining centres). Furthermore, the introduction of new part types may cause significant preparation times (NC programs, clamping plans) and loss of production time (caused by testing activities).

\section{Multifunctionality}

An FMS is capable of performing several processing steps of a job which traditionally were executed on subsequent production units. This multifunctionality can be realized in two different ways: (i) through the use of multifunctional machining centres or (ii) through the integration of various types of workstations in one system.

An effective use of an FMS requires the integration of the efficiency, flexibility and multifunctionality aspects of the system. A manufacturing planning and control system (MPCS) is a key condition to deal properly with the limitation of the efficiency and flexibility also mentioned above. Such a system is often based on a hierarchical decision structure. ${ }^{6,10,12}$ In Section 2 we discuss the basic framework of such a hierarchical planning and control system. In Section 3 we use a practical case to describe in some more detail solution approaches to problems at the subsequent levels. A brief summary and some concluding remarks are given in Section 4.

\section{FRAMEWORK OF AN MPCS DECISION HIERARCHY}

Several arguments can be given to justify a hierarchical approach to manufacturing planning and control problems, for instance:

(i) Reduction of complexity. The problem of planning and controlling a complex production system is often characterized by the presence of 
multiple, sometimes contradictory, objectives and a number of complicating and to a certain extent conflicting constraints. A hierarchical approach offers the possibility of splitting up complex interrelated production control problems into several small solvable parts.

(ii) Separation of short-, medium- and long-term aspects. Production planning and control problems on a long-term level are generally more strategic in nature than medium-term (tactical) and shortterm (operational) problems and therefore demand different solution methods. In this paper, we are mainly concerned with medium- to shortterm planning problems. For example, in the medium term, capacity planning is usually performed in terms of aggregate products (of product families) whereas in FMS loading and scheduling problems we are dealing with the actual product mix. ${ }^{5}$

(iii) Improving stability and controllability. Production control problems may arise at regular and/or irregular intervals. Without a hierarchical decision structure all the (interrelated) production control problems are affected by any disturbance. A hierarchical approach offers the possibility of solving problems on one level without the need to replan on higher levels. This improves the stability of the production planning and control decisions and severely reduces the amount of information required.

Within an MPCS decision hierarchy three main levels can generally be distinguished: (i) an assignment level, (ii) an off-line level and (iii) an on-line level (see Fig. 1). The choice of these three main levels is, to a large extent, a pragmatic one: the levels often reflect organizational practice in many companies as will be seen in the remainder of this paper.

On the assignment level jobs are distributed among the various production units, including the FMS. The assignment level is responsible for generating realizable throughput times and a realistic workload of the FMS. In many practical situations, the activities on the assignment level are executed periodically, for instance monthly or sometimes weekly. In most cases an MRP system is used at the assignment level.*

The off-line level receives jobs from the assignment level. The off-line level is responsible for getting a good fit between the received jobs and the characteristics (limitations) of the FMS. Off-line decisions are based upon a model of the FMS, in which most important characteristics (limitations) of the FMS are incorporated. In most practical situations off-line activities (if present) are again executed periodically, with a

\footnotetext{
*Within the assignment level it might be difficult to take account of the specific characteristics (or limitations) of the FMS. In an MRP system, for instance, and FMS is just seen as a capacity resource. There are few attempts to integrate FMS production planning problems into a closed-loop MRP system. ${ }^{7}$
}

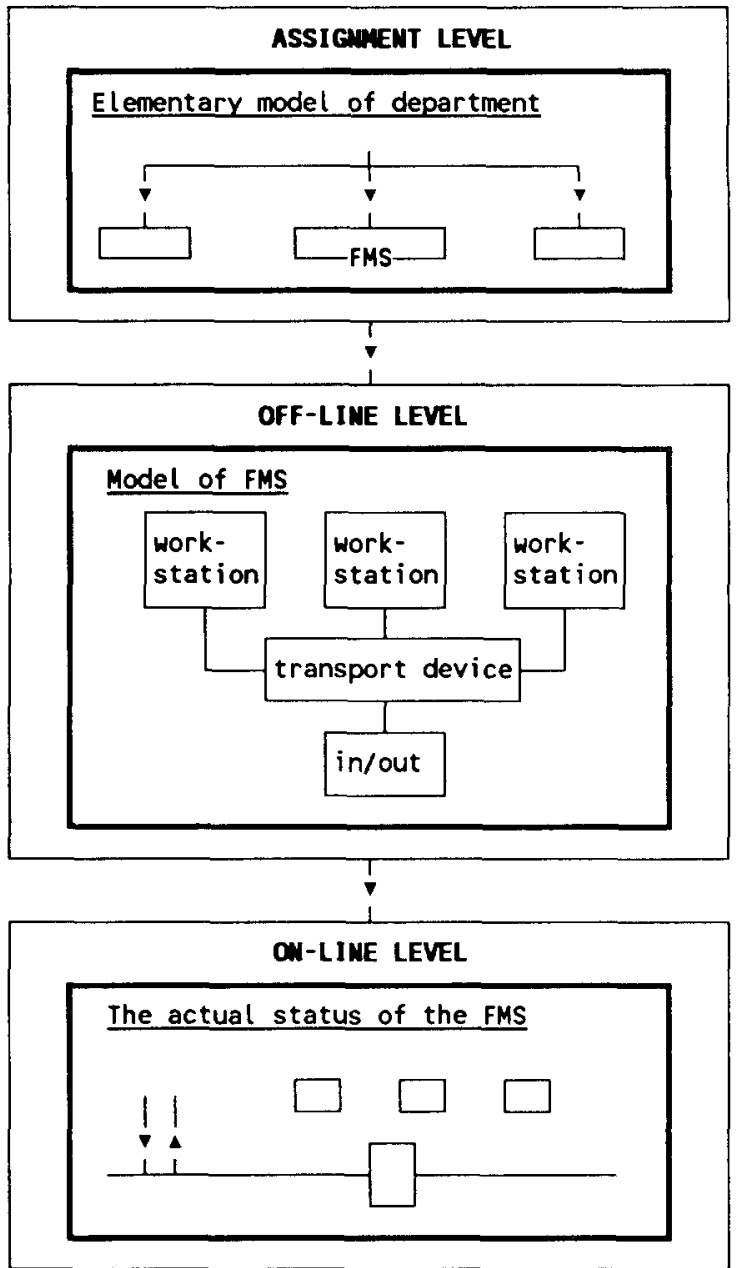

Fig. 1. Decision levels in the production control of a FMS.

smaller review period, for instance weekly or even daily. The off-line activities may concern the batching of jobs to be produced in the same period and the loading (assignment) of operations and tools to the various workstations. Sometimes, the sequencing of jobs is also carried out on the off-line level. ${ }^{9}$

On-line activities are based upon the information from the off-line level and upon the actual status of the FMS. On-line decisions can be taken at any moment. These decisions concern the release of jobs to the FMS and the sequence in which competitive activities have to be performed (dispatching). The degree of detail at the off-line level determines the degree of freedom at the on-line level.

In the above decision hierarchy we implicitly assume that jobs are completely known in terms of their processing characteristics, i.e. their process plans, specifying among other things fixtures and tools needed as well as the relevant processing times. An alternative is discussed briefly by Van Houten ${ }^{2}$ where the selection procedure for an appropriate process plan takes into account capacity balancing arguments as well as the availability of tools. Another alternative for batching, loading and release is the so-called "flexible approach $^{11}$ ", where decisions about the actual job mix are based primarily on the actual status of the 
system with the objective of optimizing certain performance measures such as machine utilization and due date performance. Basically, the flexible approach can be seen as an intelligent form of releasing.

The above three-level hierarchy can be recognized in most planning and control systems. Differences are often due to the extent to which sophisticated algorithms are applied, the location of certain subproblems to another level (for instance on-line loading) or even the complete absence of one level (e.g. the off-line level). In the next section we specify the above structure for a real life example and show how problems on the subsequent levels can be handled.

\section{MANUFACTURING PLANNING AND CONTROL: A REAL LIFE EXAMPLE}

In this section we consider a company which produces pneumatic and electric actuators for valves. Recently, the company was facing a rapid increase in both volume and mix (diversity), resulting in among other things a dramatic increase in the required metalcutting capacity. The diversity of products is to a large extent determined by the actuator housings. In an attempt to keep leadtimes relatively short, an FMS has been installed to manufacture these customerspecific housings. This has resulted in a logistic structure in which component manufacture is based on forecasts, except for the FMS operation on the housings, while assembly is also order based (see Fig. 2). For housings, the procurement of raw materials and basic component manufacturing takes about 5 weeks after which 3 more weeks are reserved for the FMS operation, some finishing operations and the final assembly (1 week each), yielding a total leadtime of 8 weeks.
The number of different housings before the FMS operation is about 15 . This corresponds with the variety of other part types needed for assembly. The number of different housings after the FMS operation is about 150. FMS constitutes the bottleneck in the system.

Figure 3 gives a schematic representation of the particular FMS considered. Three basically identical machining centres (M1, M2 and M3) are linked together by a pallet transport vehicle and an integrated pallet buffer system, having a capacity of 28 pallets. Each of the three machines can hold a maximum of 40 tools in the tool magazine. The changing of tools in the tool magazines is performed automatically. A tool robot allows the connection between a central tool storage and the tool magazines. An FMS computer takes care of the coordination of all activities within the FMS on the on-line level (compare the preceding section). The clamping and unclamping of parts on pallets is done manually on two integrated load/unload $(\mathrm{L} / \mathrm{U})$ stations.

\subsection{Manufacturing planning problems: the assignment level}

The manufacturing planning system of the firm basically operates as follows. Every quarter, a sales forecast is presented on an aggregate level (corresponding to the 15 basic types, $c f$. Fig. 2). Based upon this forecast, raw materials procurement and all component manufacturing operations, except those performed on the FMS, can be started.

The planning department translates the aggregated forecasts into a master production schedule (MPS), using only rather rough rules such as economic batch size quantities and an estimation of the required

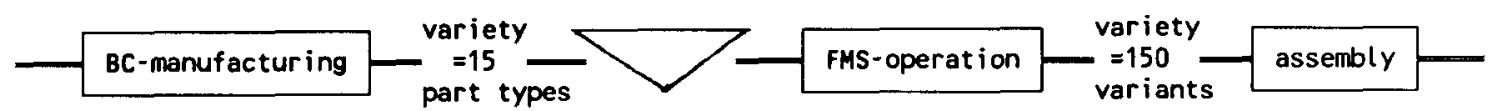

Fig. 2. Integration of FMS in the manufacturing department (BC-manufacturing = basic component manufacturing).

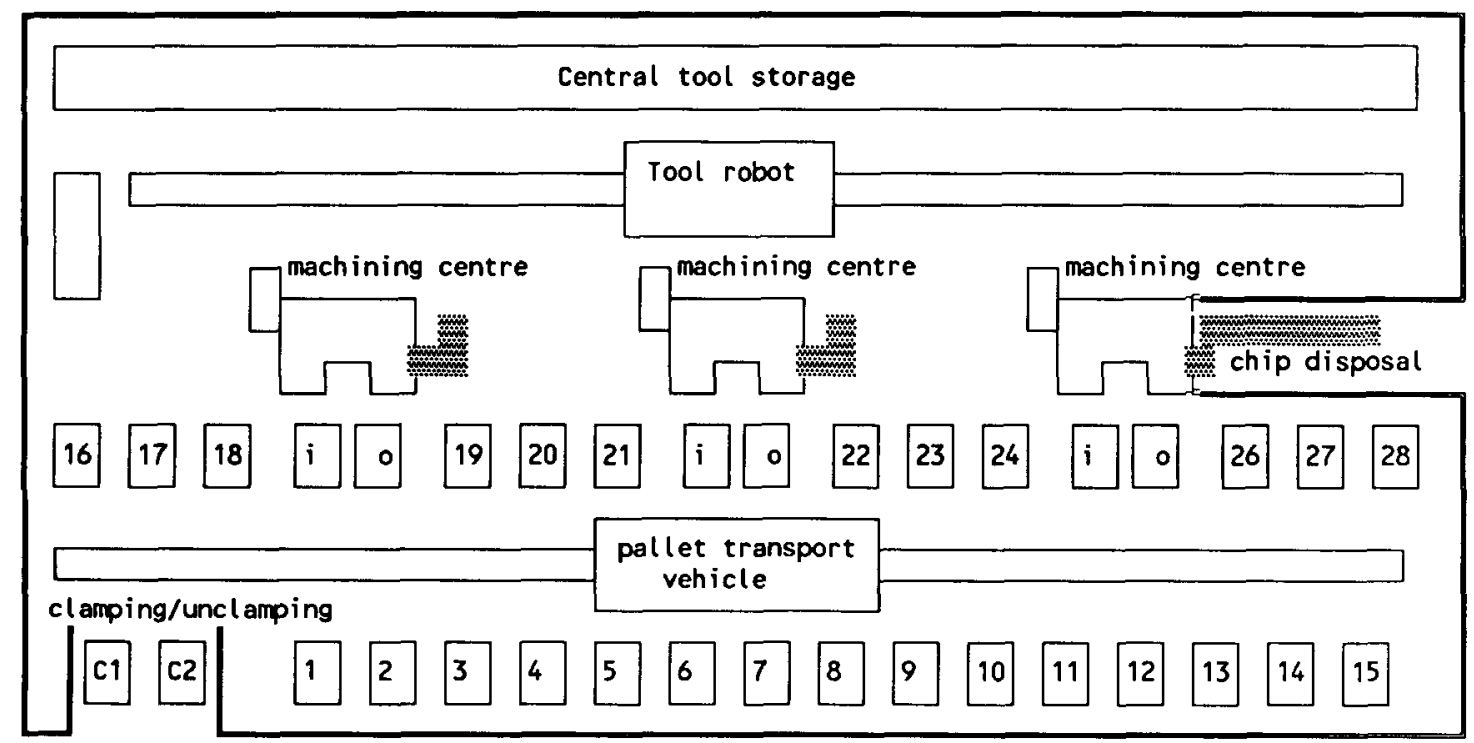

Fig. 3. Schematic representation of the FMS. 


\begin{tabular}{|l|cccccc|}
\hline Aggregate product & A & B & C & D & E & $\ldots \ldots \ldots$ \\
\hline $\begin{array}{l}\text { Forecast } \\
\text { (12 week period) }\end{array}$ & $\begin{array}{r}1200 \\
250\end{array}$ & 450 & 400 & 40 & $\ldots \ldots \ldots$
\end{tabular}

Fig. 4. Aggregated forecast of products.

(machine) capacity on the FMS (recall that the FMS constitutes the bottleneck). Figure 4 presents an aggregated forecast which, in Fig. 5, is translated into an MPS. During the first 5 weeks of the total manufacturing leadtime, customers are invited to subscribe definitively. At the end of week 5 , the subscription is frozen (or closed). In the case of a full subscription, new customers have to subscribe for a later delivery (or a subsequent batch of the same type is expedited), in case not all planned basic components are needed, some stock is left in the component store before the FMS.

\subsection{Batching, loading and release problems for the FMS}

As mentioned earlier, planning at the off-line level is done periodically, in our case weekly, where the master production schedule (MPS) discussed above yields the input for this off-line level. Unfortunately, the planning and control of the FMS is complicated by several aspects. Some of these aspects are related to the specific characteristics of the FMS, other aspects are related to the structure of the MPCS. The main aspects are:

1. In principle, each production batch is assigned to just one pallet/fixture combination. All parts of the job need this pallet/fixture (for several part types there even exists only one unique pallet/fixture combination). Therefore, in order to avoid machine idle time caused by the (sometimes severe) time needed to unload and subsequently load a pallet, batches of different jobs are processed in a so-called job-mix routine, e.g. A-B-C-A-B-C- etc. After processing job $A$, the pallet moves to the output position of the machine. The pallet at the input position will be moved into the machine. Job B can start. At that moment the input position is free. The first priority of the transport device is to avoid empty input positions. As a consequence, a new pallet out of the pallet buffer will be transported to the input position. This means that after job B, job
$C$ (if available) will be performed. During the machining of job $\mathrm{B}$, there is some time to transport the pallet in the output position to a clamping/ unclamping station and to substitute the manufactured parts ( $c f$. Fig. 6).

2. To avoid too many tool changes during the procesing of a mix of jobs (see aspect 1) it is desirable that the number of cutting tools needed for a mix is less than or equal to the capacity of the tool magazine.

3. A unique cutting tool cannot be used simultaneously on more than one machining centre.

4. A unique fixture cannot be used simultaneously for more than one job.

5. Theoretically the three machining centres are identical. However, in practice, there are some minor, but sometimes important, differences. The accuracies of the three machining centres are distinct. Furthermore, extensive chip disposal equipment is only integrated with one machining centre. These facts reduce the interchangeability of the machining centres to some extent. Some jobs have to be assigned to one specific machining centre.

6. Jobs (customer orders) have to be performed on the FMS within 1 week. This is to conform with the MPCS structure of the company.

7. The FMS is the capacity bottleneck. A high utilization of the FMS is essential. Generally, work in the shop is done in two manned shifts with a partial third unmanned shift (for the FMS). To utilize the unmanned shift as much as possible, it is desirable

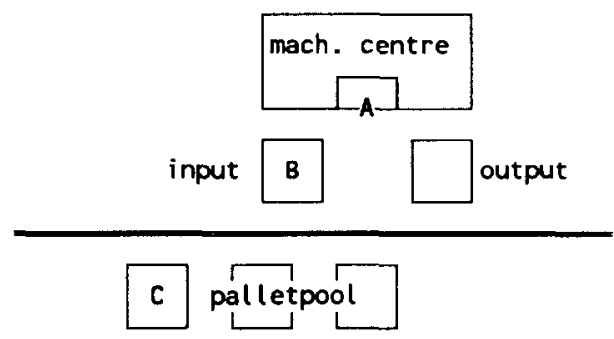

Fig. 6. Jobs/operations running in a mix.

\begin{tabular}{|c|c|c|c|c|c|c|c|c|c|c|c|c|c|}
\hline $\begin{array}{l}\text { aggre- } \\
\text { gated } \\
\text { product }\end{array}$ & 1 & 2 & 3 & 4 & 5 & time & $\begin{array}{c}\text { (week } \\
7\end{array}$ & 3) 8 & 9 & 10 & 11 & 12 & \\
\hline $\begin{array}{l}\text { A } \\
\text { B } \\
\text { C } \\
\text { D } \\
\text { E } \\
. \\
.\end{array}$ & $\begin{array}{l}50 \\
90\end{array}$ & $\begin{array}{c}300 \\
10 \\
-\end{array}$ & $\begin{array}{r}50 \\
100\end{array}$ & 90 & $\begin{array}{c}300 \\
-\end{array}$ & $\begin{array}{r}50 \\
100 \\
10\end{array}$ & - & 300 & $\begin{array}{r}50 \\
100\end{array}$ & $\begin{array}{l}90 \\
10 \\
- \\
-\end{array}$ & 300 & $\begin{array}{r}50 \\
100\end{array}$ & $\begin{array}{l}90 \\
10 \\
-\end{array}$ \\
\hline
\end{tabular}

Fig. 5. Master production schedule. 


\begin{tabular}{|cc|ccccccccc|}
\hline Variant & Tools & 1 & 2 & 3 & 4 & 5 & 6 & 7 & 8 \\
\hline 1 & & $\times$ & & $\times$ & & $\times$ & & & \\
2 & & & $\times$ & & $\times$ & & $\times$ & & \\
3 & & & & $\times$ & & $\times$ & & $\times$ & \\
4 & & & & $\times$ & & $\times$ & & $\times$ & & \\
5 & & $\times$ & & $\times$ & & & & $\times$ & \\
6 & & & & $\times$ & & & & & & \\
\hline
\end{tabular}

\begin{tabular}{|c|c|cccccccc|}
\hline Variant & Tools & 1 & 5 & 3 & 7 & 2 & 6 & 4 & 8 \\
\hline 1 & & $x$ & $x$ & $x$ & & & & & \\
3 & & & $x$ & $x$ & $x$ & & & & \\
5 & & $x$ & & $x$ & $x$ & & & & \\
2 & & & & & & $x$ & $x$ & $x$ & \\
4 & & & & & & $x$ & $x$ & $x$ & \\
6 & & & & & & $x$ & $x$ & & $x$ \\
\hline
\end{tabular}

Fig. 7. Variant/cutting_tool table, before and after rearrangement.

to have sufficient jobs (customer orders) available to fill the pallet buffer. Furthermore, operations with long processing times should preferably be assigned to the unmanned shift.

Next, we describe a few basic techniques to solve some of the problems listed above. Clustering techniques are used to group together those parts which have certain similarities in operational characteristics, resulting in a certain overlap in required tools. This becomes even more important if a large number of tools are unique (in the case discussed $57 \%$ of the cutting tools appeared to be unique). Basically, clustering boils down to the rearrangement of rows and corresponding columns in a variant/cutting_tool matrix, as illustrated below.

Many algorithms have been developed to perform the clustering (with slightly varying objectives). ${ }^{3,5,8}$ For our purposes, the algorithm of McCormick et al. ${ }^{8}$ performed best. ${ }^{1}$

Once clusters of variants (customer orders) are constructed, loading of these clusters (batches) and of the corresponding tools to the machines is usually performed by solving integer programming problems with a relatively easy structure. As an example we give the formulation which attempts to balance the load among three identical workstations, taking into account tool magazine capacity limitations:

$$
\operatorname{minimize}\left(\max _{m}\left[\Sigma_{i} t_{i} x_{i m}+\delta\left(\Sigma_{j} y_{j m}-c_{m}\right)^{+}\right]\right)
$$

under

$$
\begin{aligned}
\Sigma_{m} x_{i m} & =1 & & \text { for each cluster } i \\
y_{j m} & \geq x_{i m} & & \text { for all } j \in J(i), \text { for all } i \\
\Sigma_{m} y_{j m} & \leq b_{j} & & \text { for each tool type } j \\
x_{i m}, y_{j m} & =0,1 & &
\end{aligned}
$$

where $J(i)$ is the set of tool types necessary to process cluster $i, b_{j}$ the number of available tools of type $j, c_{m}$ the tool magazine capacity of machine $m$ and $t_{i}$ the cumulative processing time of all parts in cluster $i$. Furthermore $x_{i m}=1\left(y_{j m}=1\right)$ if cluster $i$ (tool $\left.j\right)$ is loaded on machine $m$, otherwise $x_{i m}=0\left(y_{j m}=0\right)$. In the objective function we first try to balance the workloads among the machines while a second criterion seeks a minimum overload of tool magazines (recall that some overload is possible due to the presence of the tool robot). The weight of this second criterion is controlled by the parameter $\delta$. The above formulation is easily adapted for the situation where certain jobs have to be processed on one specific machine.

Finally, at the sequencing/dispatching level one has to take into account the fact that for part types A, B and $\mathrm{C}$ to be processed in a three order mix (for example) necessary conditions are of the type

$$
\begin{aligned}
& \operatorname{LOAD}(\mathrm{A}) \leq \mathrm{PRT}(\mathrm{B})+\operatorname{PRT}(\mathrm{C}) \\
& \operatorname{LOAD}(\mathrm{B}) \leq \mathrm{PRT}(\mathrm{A})+\operatorname{PRT}(\mathrm{C}) \\
& \operatorname{LOAD}(\mathrm{C}) \leq \mathrm{PRT}(\mathrm{A})+\operatorname{PRT}(\mathrm{B})
\end{aligned}
$$

$\operatorname{LOAD}(\mathrm{A})+\operatorname{LOAD}(\mathrm{B})+\operatorname{LOAD}(\mathrm{C}) \leq$ PRT(A) + PRT(B) + PRT(C)

Where LOAD denotes the time needed to unload a pallet and subsequently load it with new parts of the same type, and PRT denotes the processing time of a pallet. Similar conditions hold for any part mix; in the particular case discussed here these conditions did not appear to be highly restrictive.

\section{SUMMARY AND CONCLUDING REMARKS}

In this paper, we have discussed a general hierarchical framework for planning and controlling a department in which a flexible manufacturing system is the bottleneck. The framework is applied to a real life example. The relevant FMS problems are discussed in some detail and several models are suggested to deal with resulting subproblems.

Future research includes a further investigation of alternative planning structures. For example, the present MRP system only makes a rough cut capacity check for the FMS, and only for machining capacity. Cutting tools, pallets and fixtures are not considered at this level although certainly the cutting tools represent severe capacity limitations. Differences between the machining centres are not taken into account (see aspect 5 in the preceding section). Also, the possible use of alternative process plans is an important subject for further study, involving again aspects of decision hierarchy. In particular, guidelines should be given when to use the possibility of choosing an alternative process plan in case of unexpected disturbances such as a machine breakdown and when to forward these problems to a higher level in the decision hierarchy. 


\section{REFERENCES}

1. Gruteke, R.: Production control of a particular FMS. Master's thesis, Laboratory of Production Management and Operations Research, Department of Mechanical Engineering, University of Twente, Feb. 1991 (in Dutch).

2. Houten, F. J. A. M. van: PART: a computer-aided process planning system. Ph.D. thesis, University of Twente, Department of Mechanical Engineering, 1991.

3. King, J. R., and Nakornchai, V.: Machine-component grouping in production flow analysis: an approach using a rank order clustering algorithm. Int. J. Production Res. 20(2): 117-133, 1982.

4. Kumar, K. R., Kusiak, A., Vannelli, A.: Grouping of parts and components in flexible manufacturing systems. Eur. J. Operational Res. 24: 387-397, 1986.

5. Kusiak, A.: Application of operational research models and techniques in flexible manufacturing systems. Eur. J. Operational Res. 24(3): 336-345, 1986.

6. Looveren, A. J. van, Gelders, L. F., Wassenhove, L. N.: A review of FMS planning models. In: Modelling and Design of Flexible Manufacturing Systems. Kusiak, A. (ed.). Amsterdam, Elsevier, 1986.

7. Mazzola, J. B., Neebe, A. W., Dunn, C. V. R.: Produc- tion planning of a flexible manufacturing system in a material requirements planning environment. Int. $J$. Flexible Manufacturing Systems 1: 115-142, 1989.

8. McCormick, W. T., Schweitzer, P. J., White, T. W. Problem decomposition and data reorganisation by a clustering technique. Operations Res. 20: 993-1009, 1972.

9. Slomp, J., Gupta, J. N. D.: An interactive tool for sheduling jobs in a flexible manufacturing environment. Working Paper LPMOR-91-1, University of Twente, Department of Mechanical Engineering, Laboratory of Production Management and Operations Research, April 1991.

10. Stecke, K. E.: Design, planning, scheduling and control problems of flexible manufacturing systems. Ann. Operations Res. : 1985.

11. Stecke, K. E. Kim, I.: A study of part type selection approaches in flexible manufacturing systems. Working Paper No. 498, University of Michigan, 1987.

12. Zijm, W. H. M.: Flexible manufacturing systems: background, examples and models. In Operations Research Proceedings 1988, Schellhaas, H. et al. (eds.). Heidelberg, Springer, 1988, pp. 142-161. 